\title{
The Sensitivity of the Epidemic Growth Rate to Weather Variables, with an Application to Yellow Rust on Wheat
}

\author{
Konstantina Papastamati and Frank van den Bosch
}

Biomathematics and Bioinformatics Division, Rothamsted Research, Harpenden, Herts. AL5 2JQ, UK. Accepted for publication 13 July 2006.

\begin{abstract}
Papastamati, K., and van den Bosch, F. 2007. The sensitivity of the epidemic growth rate to weather variables, with an application to yellow rust on wheat. Phytopathology 97:202-210.

We first show how to estimate the exponential epidemic growth rate, $r$, for different combinations of three weather variables. Then we derive a method to quantify the sensitivity of $r$ to a weather variable as a function of the pathogen life cycle variables of latent period, basic reproductive number, and the mean and standard deviation of the sporulation curve.

and pathogen life cycle component in terms of epidemic progress. The method is applied to yellow rust, caused by Puccinia striiformis, on winter wheat. We conclude that the most important weather variable for the progress of yellow rust is temperature, followed by dew period and light quantity. By far, the most important pathogen life cycle component is the basic reproductive number, especially at low and high temperatures. This disagrees with the general view that latent period is the most important variable at low temperatures. We discuss explanations of this.
\end{abstract} The method can be used to identify the most important weather variable
Additional keywords: exponential growth rate, sensitivity analysis.
It is well known that weather plays a key role in the development of most plant disease epidemics, with temperature, leaf wetness duration, and light being the most-studied weather variables in this context. One example is yellow rust (caused by Puccinia striiformis) on wheat (Triticum aestivum), of which the dependence of various life cycle characteristics on temperature, wetness duration, and light intensity and quantity have been studied $(3,4,7)$. These studies show the dependence of the latent period, sporulation, infection efficiency, and basic reproductive number of yellow rust epidemics on weather variables, but do not show how these effects of the weather feed through to epidemic development.

Models are frequently used in plant disease epidemiology. The route usually followed is to study the relationship of weather and disease using weather variables as the input to estimate their effect on the pathogen's life cycle variables (latent period, sporulation, infectious period, and infection efficiency) and then based on these, calculate the epidemic progress as the output. There are various types of models ranging from simple equations, like the Vanderplank model (19), the Zadoks model (26), which has been the basis for other models in plant disease epidemiology, or the SEIR models (20), to complex (simulation) models, such as those for epidemics of apple scab (23) and light leaf spot on winter oilseed rape (8). Even though these models perform well, it is difficult to compare their results because of different inputs, complexity, and different quantifications of epidemic development. Moreover, these models only show how a change in, for example, temperature works out at the epidemic level with a resulting change in disease severity. They do not, however, allow identification of the pathogen life cycle characteristic through which this change in temperature affected the epidemic the most. The difficulty in comparing existing models, and the inability of existing models to further unfold the chain of

Corresponding author: F. van den Bosch;

E-mail address: frank.vandenbosch@bbsrc.ac.uk

DOI: 10.1094/PHYTO-97-2-0202

(C) 2007 The American Phytopathological Society cause and effect relations between weather variables, pathogen life cycle variables, and epidemic progress shows the need for a standardized systematic method to this end. We present the development of such a method in this paper.

In the initial stages of an epidemic most plants and plant parts are susceptible and density dependence is negligible. In this phase the epidemic grows exponentially with growth rate, $r$. In continuous time, $d X / d t=r X$ with solutions of $X=X(0) e^{r t}$ where $X$ is the pathogen density and the initial condition is $X(0)$, represents exponential growth or decline with growth when $r>0$ and decline when $r<0$ (6). We use here the term "epidemic" to signify the pathogen and not the visual symptoms of disease. The exponential growth rate, $r$, depends on the pathogen's life cycle components, i.e., the latent period, the infectious period, the sporulation curve, and the basic reproductive number and hence, on any weather variable that might affect these.

The basic reproductive number is defined as the total number of new daughter lesions produced by a mother lesion in the early stages of an epidemic. Following Segarra et al. (14) we define the basic reproductive number as

$$
R_{0}=\psi \xi H_{0} \int_{0}^{\infty} I(\tau) d \tau
$$

with $\xi$ the probability that a spore is deposited on a healthy leaf, $\psi$ the probability that a spore deposited on a healthy leaf will initiate an infection, $I(\tau)$ the sporulation curve, and $\tau$ the age of infection, i.e., $\tau$ shows when an infection was caused (14). $I(\tau)$ is defined as the number of spores produced per unit of time by an infected site of age $\tau$, taking into account both the latent $[I(\tau)=0]$ and the sporulation $[I(\tau)>0]$ period. The integral gives the total number of spores produced until the end of the sporulating (infectious) period.

Recently, Segarra et al. (14) discussed that a range of models, including the Vanderplank and SEIR models, are special cases of the Kermack and McKendrick model. For low pathogen density, the Kermack and McKendrick model reduces to a linear form (14), and the exponential growth rate, $r$, can be calculated from the Euler equation: 


$$
1=R_{0} \int_{0}^{\infty} e^{-r \tau} i(\tau) d \tau
$$

where $R_{0}$ is the basic reproductive number, and $i(\tau)$ is the normalized sporulation curve (14). A summary explaining the derivation of equation 2 is given in Appendix 1. The initial exponential growth rate, $r$, can therefore be estimated for any type of model that is a special case of the Kermack and McKendrick one. Since the exponential growth rate is strongly influenced by weather variables and is a parameter widely used to describe epidemics, it would be useful to have methods to (i) calculate $r$ from the Euler equation as a function of weather variables and (ii) quantify how the effect of weather variables on specific pathogen life cycle components is passed through to $r$. It then becomes possible to compare different weather scenarios for the same pathogen, or different pathogens under the same weather conditions. Such a method would also provide insight into how the progress of an epidemic is influenced by weather variables and in doing so, provide suggestions for disease management. In this paper we derive such a method using the ideas of sensitivity analysis as described by Caswell (1), and apply it to the example of yellow rust on wheat. Sensitivity analysis quantifies, in our case, the response of the exponential growth rate of the epidemic, $r$, to changes in a weather variable. Sensitivity analysis became popular with the development of matrix population models, but no comparable method has been developed for the Euler equation and the models it represents (13).

In this paper, we first show how to calculate the exponential growth rate when the basic reproductive number, $R_{0}$, and the spore production curve, $i(\tau)$, depend on weather variables. Then, a method is developed to calculate the sensitivity of the exponential growth rate to weather variables. After the theoretical description of the methodology, published data on yellow rust are used to demonstrate (i) the type of information needed to apply this method, (ii) the ease of application and, most importantly, (iii) the output produced and how this can facilitate the unraveling of the relationship between weather and disease.

\section{THEORY AND APPROACHES}

Generic methods. Exponential growth rate. Several authors have shown that the sporulation curve, $i(\tau)$, is well described by a delayed gamma distribution function $(5,7,18)$ (Fig. 1). The delayed gamma distribution is delayed by the latent period, $p$, after which spore production starts increasing gradually from zero at $\tau=p$ to reach a peak value and then decreasing back to zero at the end of the sporulation period (Fig. 1). The spore production curve is described by the mean and standard deviation ( $\mu$ and $\sigma$, respectively) of the gamma distribution, with $\mu$ the mean time

\section{Maris Beacon Maris Nimrod Muntsman}
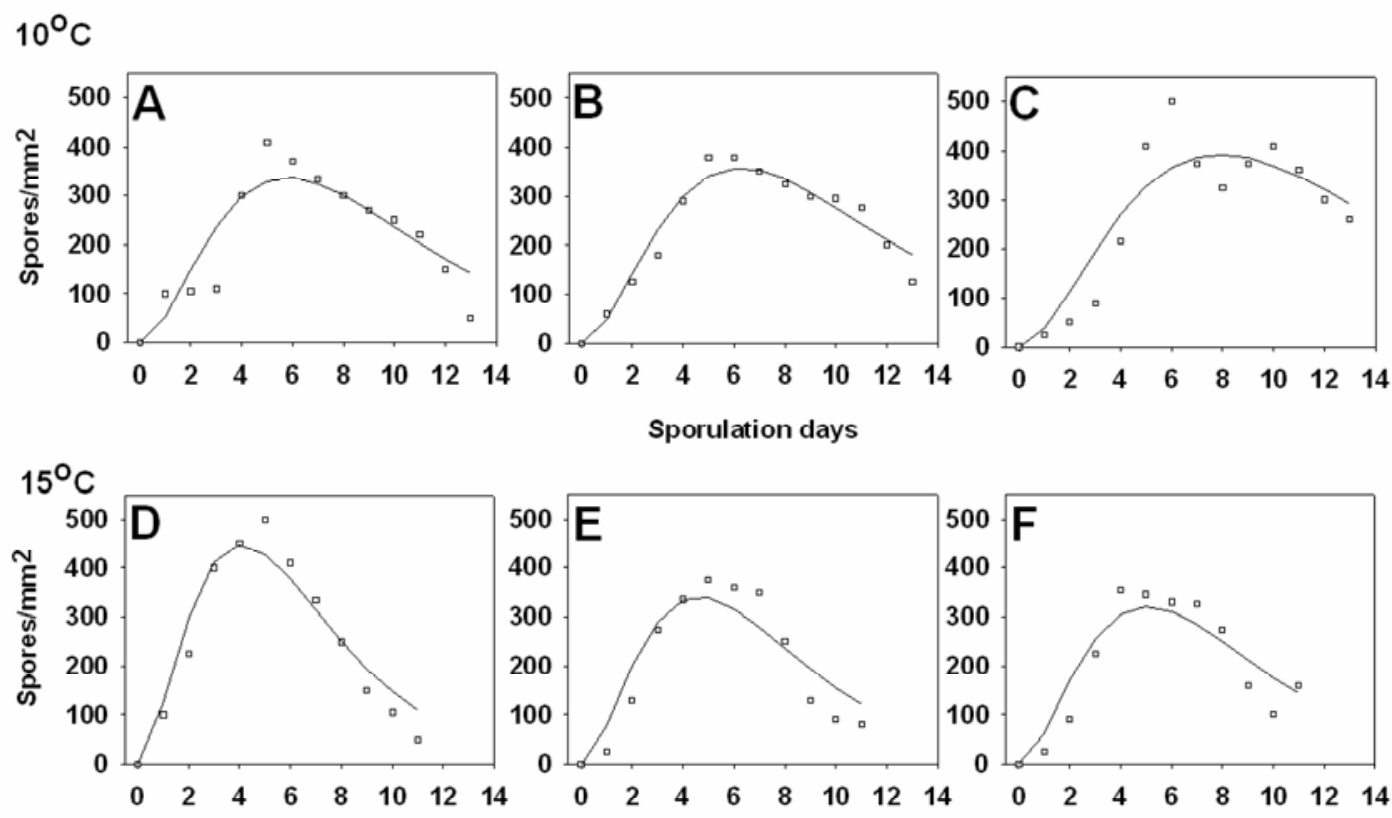

$20^{\circ} \mathrm{C}$

Sporulation days
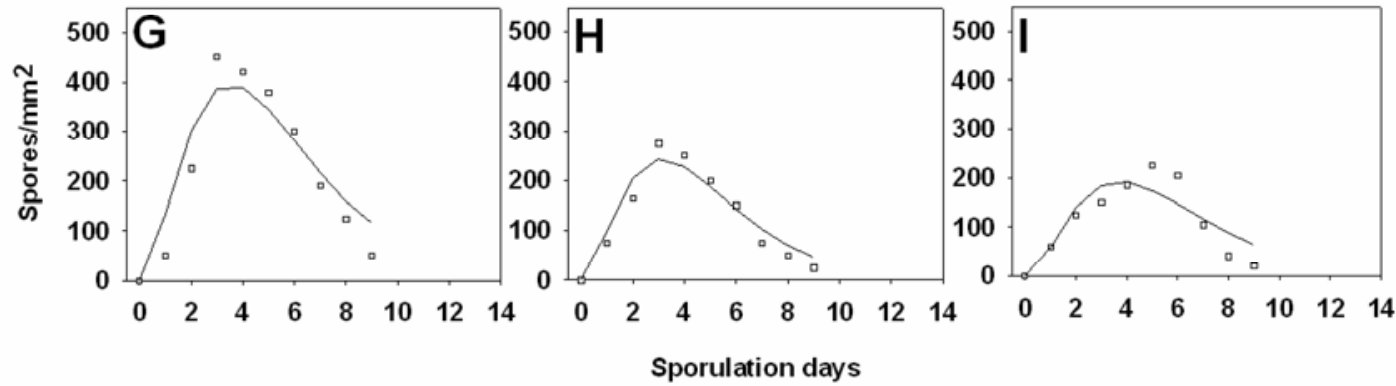

Fig. 1. Spore production data of yellow rust (Puccinia striiformis) found in McGregor and Manners (7), per square millimeter of infected leaf area ( $y$ axis) for each day after the end of the latent period ( $x$ axis), on three wheat cultivars (Maris Beacon, Maris Nimrod, and Maris Huntsman) at 10,15 , and $20^{\circ} \mathrm{C}$. The solid lines are the fitted sporulation curves, i.e., $I(\mathrm{t})$ in Appendix 2. 
after the end of the latent period $p$, of producing a spore; $\sigma^{2}$ represents the variation of this time. Using this type of sporulation curve, the Euler equation (equation 1) becomes (14)

$$
R_{0}(x) e^{-r(x) p(x)}\left[\frac{1}{\left(r(x) \sigma^{2}(x) / \mu(x)\right)+1}\right]^{[\mu(x) / \sigma(x)]^{2}}=1
$$

The latent period, $p$, mean, $\mu$, and standard deviation, $\sigma$, of the spore production distribution, and the basic reproductive number, $R_{0}$, are basic characteristics of the pathogen life cycle. Their dependence on weather is represented by setting $R_{0}, p, \mu$, and $\sigma$ as functions of $x$, which represents weather and can be one or more weather variables. Equation 3 cannot be solved explicitly for $r$. Various software packages with root-finding algorithms can be used to calculate $r$ for any combination of the weather variables, $x$. In our application of this method, $x$ is any combination of temperature $(T)$, dew period $(D P)$, and light quantity $(L)$.

Sensitivity analysis. As mentioned in the introduction, sensitivity analysis quantifies the response of the exponential growth rate, $r$, to changes in weather variables. This sensitivity is calculated by the derivative of the exponential growth rate with respect to each of the weather variables under consideration, $x$. In Appendix 2 we show how this derivative is given by the equation

$$
\frac{\partial r}{\partial x}=-\left[\left(\frac{\partial r}{\partial R_{0}} \frac{\partial R_{0}}{\partial x}\right)+\left(\frac{\partial r}{\partial p} \frac{\partial p}{\partial x}\right)+\left(\frac{\partial r}{\partial \sigma} \frac{\partial \sigma}{\partial x}\right)+\left(\frac{\partial r}{\partial \mu} \frac{\partial \mu}{\partial x}\right)\right]
$$

This expression is our key result. It unravels the effect of weather on the exponential growth rate, $r$, through the various pathogen life cycle components. If we take the first product on the right side of equation 4 , the sensitivity of $R_{0}$ to $x$ is multiplied by the sensitivity of $r$ to $R_{0}$. So the change in $R_{0}$ after a unit change in the weather variable, $x$, is multiplied with the change that will happen to $r$ after a unit change in $R_{0}$. This product shows the change in $r$ after a unit change in $x$ through the effect this change in $x$ first had on $R_{0}$. Therefore, the sensitivity of $r$ to changes in the weather variable, $x$, is the sum of the sensitivity of $r$ to the weather variable, $x$, through its effect on the life cycle characteristics $R_{0}, p$, $\sigma$, and $\mu$. So not only can we calculate the sensitivity of the exponential growth rate, $r$, to the weather variable, $x$, but we can quantify the effect of the weather on $r$ through each of the life cycle components and therewith assess whether it is the effect of the weather on the life cycle component or the effect of the life cycle component on the exponential growth rate that is the more important.

An application to yellow rust. Data sources. We have used published data from McGregor and Manners (7) and de Vallavieille-Pope et al. (3) to study the effect of temperature, $T$, dew period, $D P$, and light quantity, $L$, on the exponential growth rate of epidemics of yellow rust caused by $P$. striiformis. McGregor and Manners (7) conducted glasshouse experiments to study the sporulation rate per unit area of leaves covered uniformly with pustules under three different temperatures (10, 15 , and $20^{\circ} \mathrm{C}$ ). They also studied the effect of temperature on latent period using the same three temperatures. de VallavieillePope et al. (3) studied the effect of postinoculation temperature and dew duration on infection in controlled environments, and the effect of preinoculation light intensity on infection in the field and under controlled conditions. They assessed infection by counting the number of sporulating pustules, while they calculated infection efficiency as the number of chloroses per unit leaf area and spore. In order to use this information we define the latent period, $p$, the mean, $\mu$, and the standard deviation of the sporulation curve, $\sigma$, and the basic reproductive number, $R_{0}$, as functions of weather variables in the following sections. All the curve fittings described below were done using nonlinear least square regression.

Latent period and sporulation. The effect of temperature on the latent period, $p$, of $P$. striiformis infecting wheat cvs. Maris Beacon, Maris Nimrod, and Maris Huntsman was studied by McGregor and Manners (7) under controlled conditions. Their
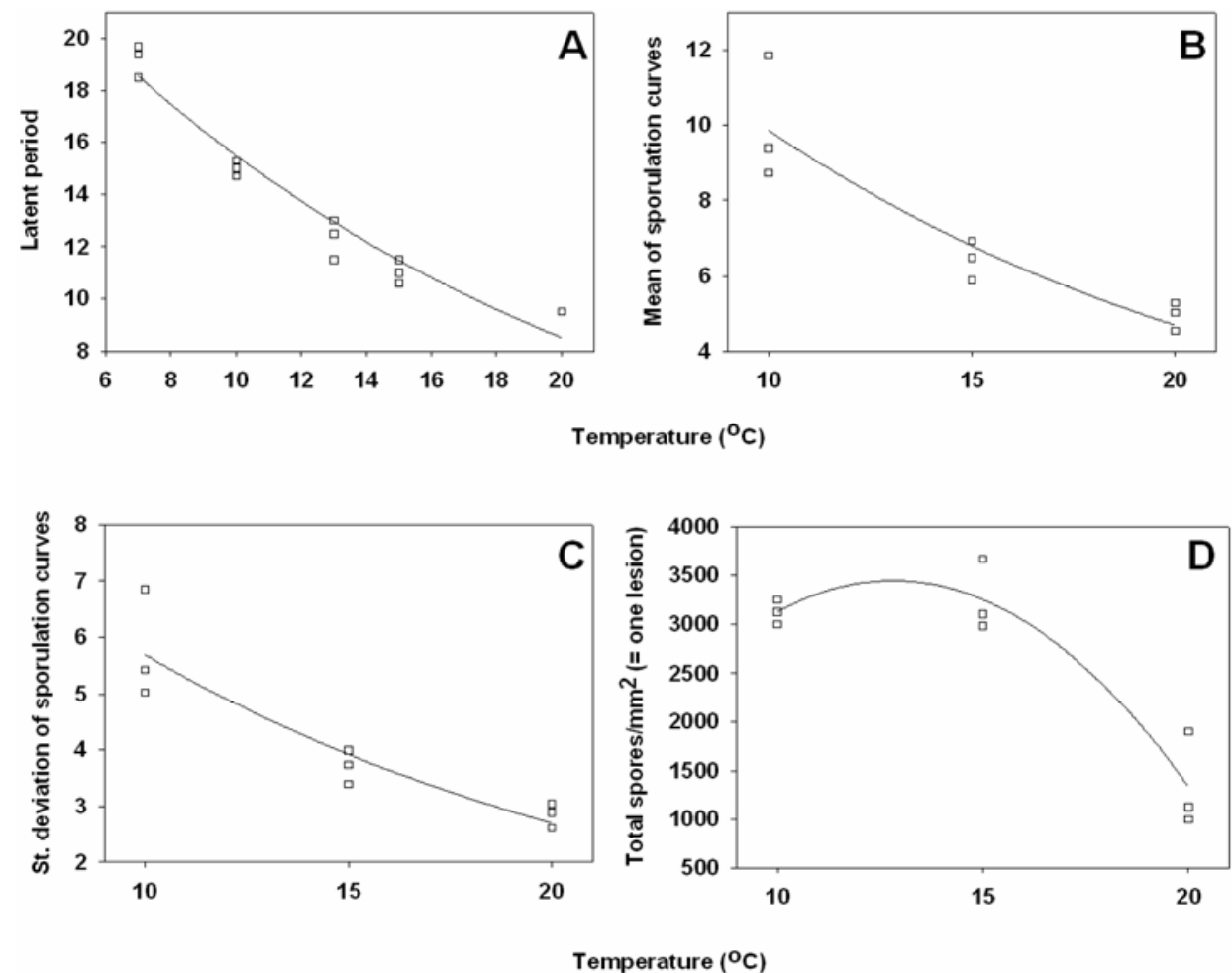

Fig. 2. Estimated functions of temperature for yellow rust (Puccinia striiformis) shown in Fig. 1 and describing A, the latent period; $\mathbf{B}$ and $\mathbf{C}$, the mean and standard deviation of the gamma sporulation curves; and $\mathbf{D}$, total spore production per square millimeter of infected leaf area. The mean and standard deviation of a gamma curve with parameters $n$ and $\lambda$ are $\mu=n / \lambda$ and $\sigma=\sqrt{ } n / \lambda$. The data for the latent period and total spore production are from McGregor and Manners (7). 
results agreed well with those of Zadoks (25). An exponential curve relating temperature to latent period, which ranged between 9 and 20 days, was fitted to the data (Fig. 2A, Table 1). The effect of temperature on spore production rates was studied by McGregor and Manners (7) on wheat cvs. Maris Beacon, Maris Nimrod, and Maris Huntsman at temperatures of 10, 15, and $20^{\circ} \mathrm{C}$. Their data were used to fit a sporulation curve to each combination of cultivar and temperature, as explained in Appendix 1. The interpolated data points and the fitted curves are shown in Figure 1. The mean, $\mu$, and the standard deviation, $\sigma$, of the fitted curves were not significantly different between cultivars, and exponential functions of temperature were fitted to describe these (Fig. 2B and C, Table 1).

Basic reproductive number. McGregor and Manners (7) also measured the effect of temperature on the total spore production per unit of infected leaf area, which we denote $f(T)$. A quadratic function was used to describe the total spore production as a function of temperature (Fig. 2D, Table 1). The basic reproductive number is calculated as the product of the number of spores produced by one unit of infected leaf area during its lifetime, $f(T)$, the per spore probability of landing on a healthy leaf, $\alpha$, and the probability of this spore causing an infection (the infection efficiency), IE. Area is not included as such in this equation but McGregor and Manners (7) scaled sporulation to one unit of infected leaf area, so essentially we assume that this is equal to one lesion. The weather dependence of $R_{0}$ is determined through the spore production and the infection efficiency, $I E$. de Vallavieille-Pope et al. (3) derived the following function of temperature, light quantity, and dew period for the infection efficiency, $I E$, of yellow rust:

$$
I E(T, L, D P)=I E_{\max } \operatorname{RIE}(T) \operatorname{RIE}(L) \operatorname{RIE}(D P)
$$

where $I E(T, L, D P)$ is the infection efficiency at temperature, $T$, light quantity, $L$, and dew period, $D P$. $R I E(T), R I E(L)$, and $R I E(D P)$ are the relative infection efficiencies in relation to each weather variable separately, with $T$ between 2.37 and $19.8^{\circ} \mathrm{C}, L$ between 0 and $60 \mathrm{~mol}$ quanta $\mathrm{m}^{-2}$, and $D P$ between 0 and $18 \mathrm{~h}$, while $I E_{\max }$ is the maximum infection efficiency they estimated. The functions for the relative infection efficiency for each climatic variable are shown in Table 1.

We, thus, get

$$
R_{0}=\alpha f(T) I E=\alpha f(T) I E_{\max } \operatorname{RIE}(T) \operatorname{RIE}(L) \operatorname{RIE}(D P)
$$

The only remaining parameter to be estimated in equation 6 is $\alpha$, the fraction of spores that are deposited on a leaf and cause an infection. This parameter is very difficult to estimate and no convincing values can be obtained from published data. To find a suitable value for $\alpha$, we had to use an indirect method. Given estimated values of the epidemic growth rate, $r$, of yellow rust epidemics, we used the Euler equation (13) to find values of $\alpha$ that reproduced these values of $r$. Young et al. (24) reported $r$ values of 0.17 and 0.18 , Paveley et al. (9) reported 0.018 and 0.074, while van den Bosch et al. $(16,17)$ found 0.11, 0.07, 0.10, and 0.12 . These papers do not provide estimates of temperature, leaf wetness duration, or light quantity, but all experiments were done in the early summer months. We assumed that temperatures were above $8^{\circ} \mathrm{C}$ but not more than $19^{\circ} \mathrm{C}$ so that we could calculate the infection efficiency due to temperature. A value of 0.02 for $\alpha$ could reproduce such $r$ values and we used this in our calculations.

\section{RESULTS}

Exponential growth rate. Using the relationship between the weather variables (temperature, dew period, and light quantity) and the life cycle characteristics of yellow rust (basic reproductive number, latent period, mean and standard deviation of the sporulation curve) in combination with equation 3 , we constructed Figure 3. The contour lines for equal values of $r$ as functions of temperature and dew period are U shaped (Fig. 3A to C). The contour lines for $r=0$ clearly show that at low

TABLE 1. Functions of temperature fitted to data from McGregor and Manners (7) on the sporulation and latent period of yellow rust on winter wheat and components of the infection efficiency of yellow rust on winter wheat as described by de Vallavieille-Pope et al. (4)

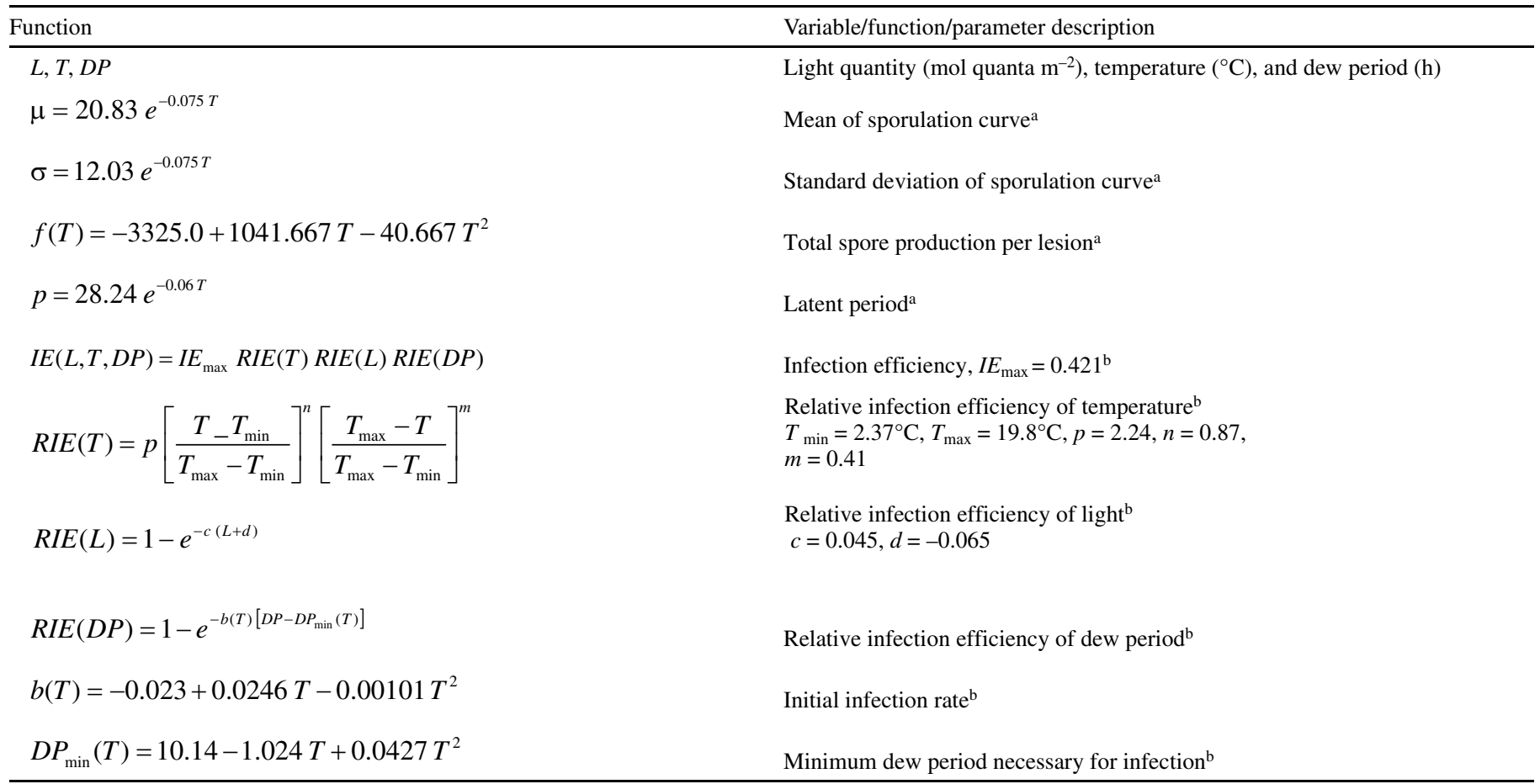

${ }^{a}$ McGregor and Manners (7).

${ }^{\mathrm{b}}$ de Vallavieille-Pope et al. (4) 
temperatures $\left(T<6\right.$ to $\left.8^{\circ} \mathrm{C}\right)$ as well as at high temperatures $(T>$ 16 to $20^{\circ} \mathrm{C}$ ), depending to some extent on dew period and light quantity, the pathogen population decreases $(r<0)$. An exponential growth rate of zero, meaning no change in pathogen density, corresponds to a basic reproductive number of one because every lesion just replaces itself so that the epidemic is sustained with no increase or decrease in pathogen density. Combining equation 6 with Table 1, we have therefore found an explicit, though complicated, invasion criterion of a yellow rust epidemic dependent on weather variables (Fig. 3A to C).

The exponential growth rate reaches its maximum at temperatures roughly between 14 and $16^{\circ} \mathrm{C}$, and decreases at higher temperatures. The latent period and the mean of the sporulation curve are decreasing functions of temperature. Either a shorter latent period, an earlier start of sporulation, both factors, or only one of them, should increase the epidemic growth rate. Therefore, the fact that the exponential growth rate, $r$, decreases at higher temperatures must be entirely due to the decreased basic reproductive number at higher temperatures $\left(T>14^{\circ} \mathrm{C}\right)$.

Lower temperatures translate into longer latent periods and delayed sporulation. These both lead to a reduced epidemic growth rate. Additionally, the basic reproductive number is small at lower temperatures, which also leads to small values for the exponential growth rate. Therefore, it is not clear which life cycle characteristic is most responsible for the low $r$ values at low temperatures. Only our sensitivity analysis will reveal which life cycle component is of key importance here.

The contour lines of the exponential growth rate as a function of dew period and light quantity (Fig. 3D to F) show the shape of a response surface for two limiting factors. Starting from low light quantity, an increase has a significant effect on the exponential growth rate, while the opposite is true when we start at a high light quantity. Therefore, light quantity is limiting at low but not at high values. The same is true regarding dew period. The combination of these two variables, which are limiting at low values, results in contours that are steep at low values of dew period and light quantity and almost horizontal at high values of dew period and light quantity.

Sensitivity analysis. The sensitivity of the exponential growth rate of the epidemic to the weather variables is shown in Figure 4. All the life cycle characteristics are affected by temperature (Fig. 4A to D), while only the basic reproductive number is affected by dew period or light quantity (Fig. 4E and F). Figure 4 clearly shows that the sensitivity of the exponential growth rate to changes in temperature is by far the greatest through its effect on the basic reproductive number. This is especially true for temperatures at which the exponential growth rate is small or even negative. The sensitivity is influenced by the other weather variables only to a smaller extent. It is also evident that the sensitivity of $r$ to the mean of the sporulation curve is greater than to its standard deviation. The sensitivities to temperature through the latent period and mean of the sporulation curve (Fig. 4A and B) are very similar and comparable to the sensitivity through the basic reproductive number at intermediate temperatures $\left(9\right.$ to $\left.15^{\circ} \mathrm{C}\right)$. The sensitivities of $r$ to the latent period and the mean of the sporulation curve are influenced by changes in dew period and light quantity.

Overall, the sensitivity of the exponential growth rate to changes in dew period or light quantity is greatest for short dew periods and low light quantity, respectively, and least when compared with the sensitivity due to temperature. This is in agreement with the shape of the contour lines for the exponential growth rate (Fig. 3D to F).

To study the sensitivity of the exponential growth rate to temperature through its effect on the basic reproductive number in more detail, we plotted the two components from equation 3 in Figure 5. This figure shows that the sensitivity of the exponential
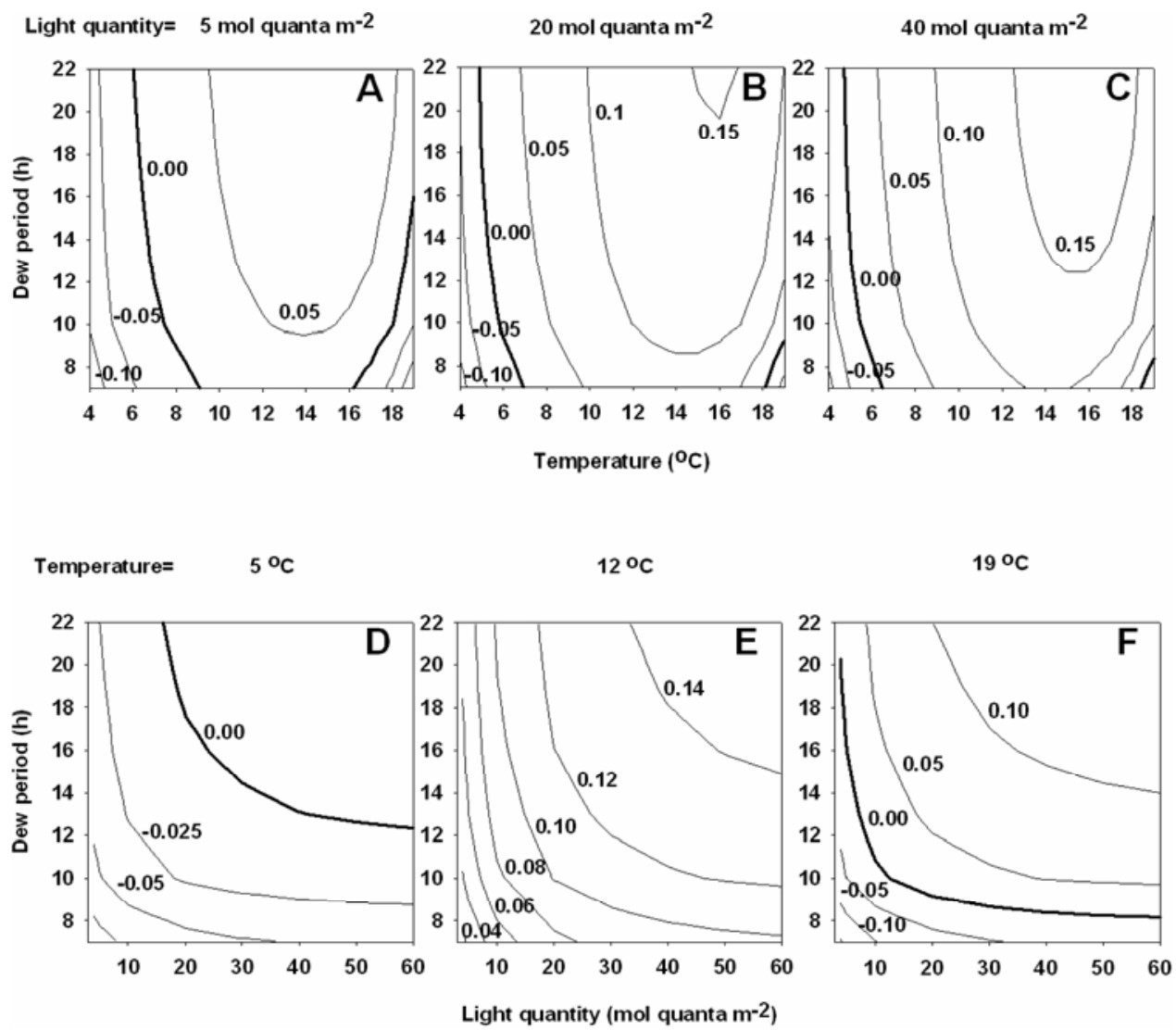

Fig. 3. Contours of estimations of the exponential growth rate, $r$, for different combinations of climatic variables. A, B, and $\mathbf{C}$ show estimates of $r$ for various combinations of dew period and temperature at set values of light quantity $\left(5,20\right.$, or $40 \mathrm{~mol}$ quanta $\mathrm{m}^{-2}$, respectively). D, E, and $\mathbf{F}$ show estimates of $r$ for various combinations of dew period and light quantity at set values of temperature $\left(5,12\right.$, or $19^{\circ} \mathrm{C}$, respectively). 
growth rate to changes in the basic reproductive number, $\partial r / \partial R_{0}$, is small compared with the sensitivity of the basic reproductive number to temperature, $\partial R_{0} / \partial x$, where $x=T$. This implies that the significance of the temperature for the exponential growth rate is mainly determined by the effect of temperature on the basic reproductive number, except at low temperatures.

\section{DISCUSSION}

The method of sensitivity analysis developed in this paper quantifies the effect of climatic variables on the exponential growth rate of epidemics in two steps. We first quantify the effect of the weather variable on the pathogen life cycle component and then the effect of the life cycle component on the epidemic growth rate. The advantage of this technique is that one can assess the sensitivities of the epidemic growth rate to a life cycle component and of the life cycle component to a climatic variable separately, and then combine them to yield the sensitivity of the epidemic growth rate to the climatic variable in question. Here, the method has been applied to yellow rust on winter wheat, and we have shown that the most important weather variable for the progress of yellow rust is temperature, followed by dew period and light quantity. Chen (2) stresses that the development of yellow rust is more sensitive to weather variables (moisture, temperature, and wind) than other plant pathogens, and that temperature is probably the most important weather variable in terms of how many pathogen life cycle components can be affected by it. We also found that the epidemic growth rate, $r$, is most sensitive to the basic reproductive number, $R_{0}$. The latter contradicts the general belief that epidemic development is mainly delayed at low temperatures by the long latent period of the pathogen. At low temperatures latent period can increase to as many as 120 days, and even up to 150 under the protection of snow cover (25). Longer latent periods might be deceptive regarding epidemic assessment and management, due to the delay of symptom appearance and lack of fungicide application on time. For example, at low temperatures, when latent periods will be longer, treatments against Septoria tritici on wheat are probably better applied earlier in the season before symptom appearance (21). West et al. (22) have reported that while phoma leaf spots on oilseed rape caused by Leptosphaeria maculans (stem canker) can be seen more or less throughout winter in Europe, in Canada,
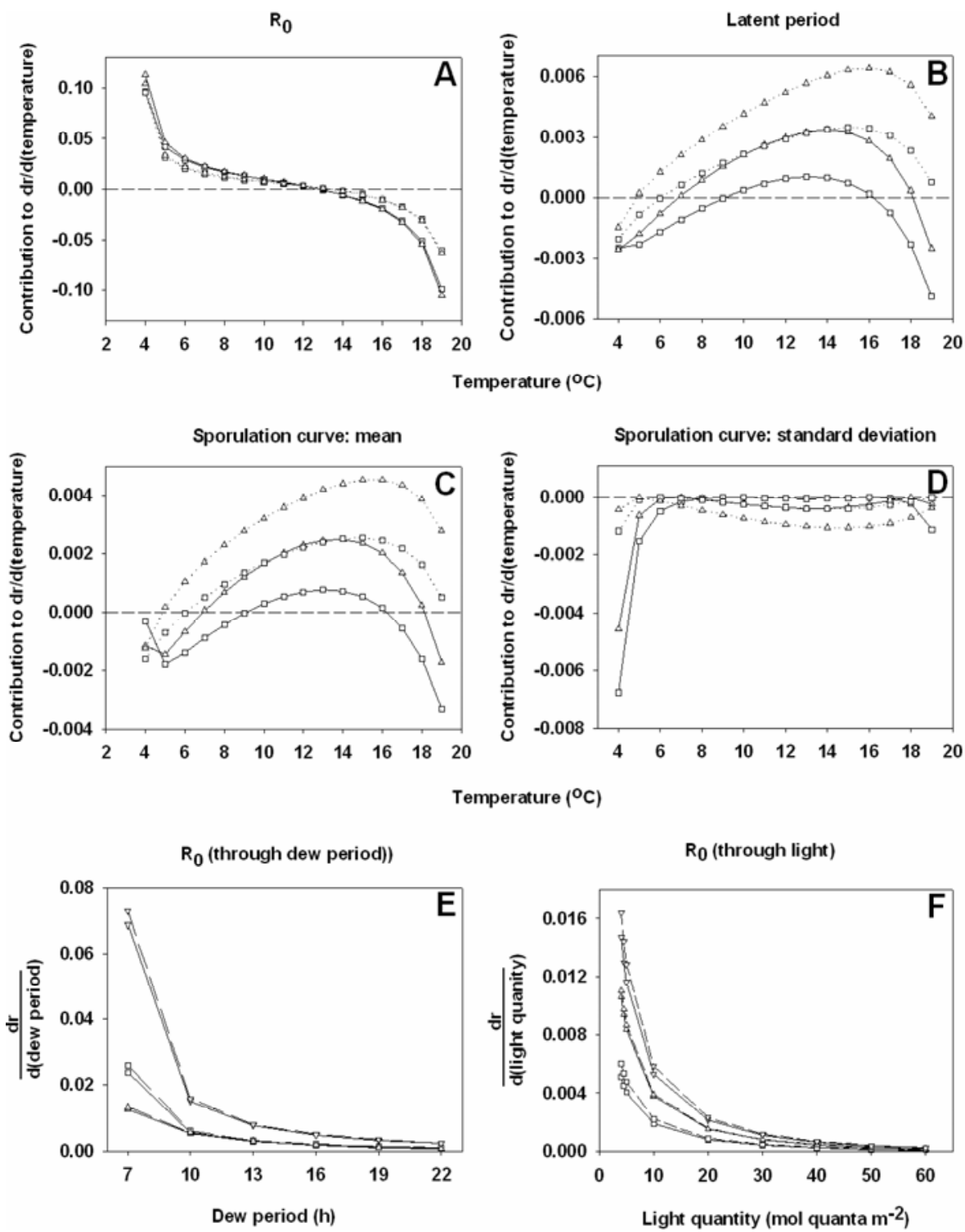

Fig. 4. Contribution of pathogen life cycle variables to the sensitivity of the exponential growth rate to $\mathbf{A}$ to $\mathbf{D}$, temperature for three dew periods (- - , - - - , and

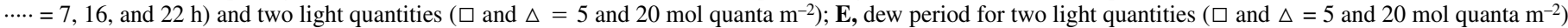
and three temperatures $\left(\square, \Delta\right.$, and $\nabla=5,12$, and $19^{\circ} \mathrm{C}$ ); and $\mathbf{F}$, light quantity for two dew periods ( $\square$ and $---=7$ and $16 \mathrm{~h}$ ) and three temperatures ( $\square, \Delta$, and $\nabla=5,12$, and $19^{\circ} \mathrm{C}$ ). 
where temperatures are lower, appearance of leaf symptoms can be delayed. Latent period has generally been studied more as it is easier to measure under different conditions of temperature, while the basic reproductive number is a more complex variable and more difficult to measure. Furthermore, the latent period is essentially a measure of the time lapse between successive generations of sporulating lesions, while the basic reproductive number is a measure of the performance of a sporulating lesion because it incorporates infection efficiency, which under specific weather conditions might compensate for a lower spore production.

The parameter $\alpha$ was chosen using an indirect method because of lack of information and because reported values of the epidemic growth rate, $r$, for yellow rust were not accompanied by observations of weather variables. Further research is needed to estimate this parameter more accurately. We note, however, that other values of $\alpha$ do not change our results qualitatively, although quantitative differences are found.

There are a few issues that may have had an effect on the results presented here, even though this effect would be quantitative rather than qualitative. The sporulation data we used refer to one unit of infected leaf area $\left(1 \mathrm{~mm}^{2}\right)$ and not to one unit of sporulating area or one lesion. However, in their experiments, McGregor and Manners (7) inoculated over the whole adaxial leaf surface and only used leaves with a uniform distribution of pustules, so that when they looked into the total spore production they referred to a unit of colonized leaf area. Likewise, we have
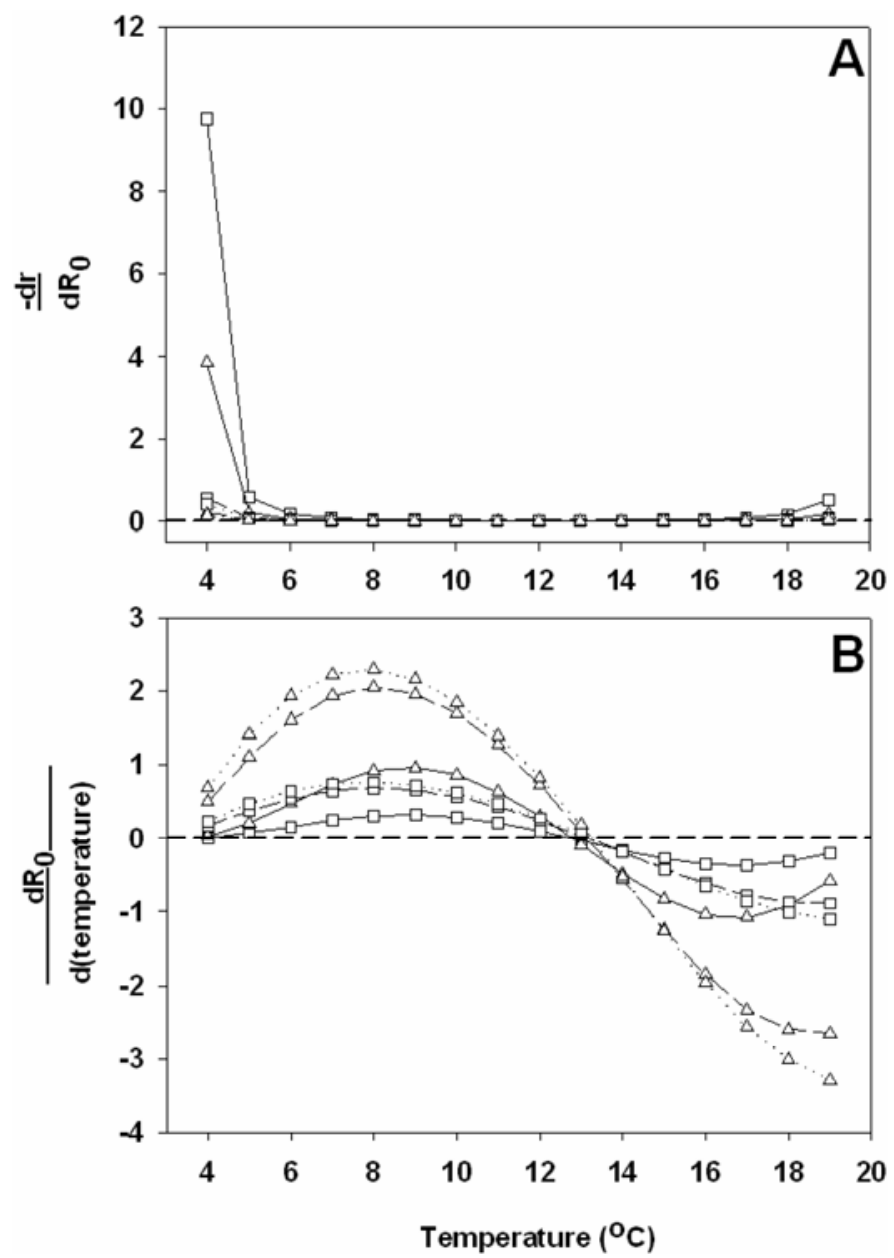

Fig. 5. Contribution of the basic reproductive number, $R_{0}$, to the sensitivity of the exponential growth rate, $r$, to temperature (Fig. 4A) is split into $\mathbf{A}$, the direct contribution of the life cycle variable, $R_{0}$, to $r$, and $\mathbf{B}$, the direct contribution of the climatic variable to $R_{0}$ for three dew periods (,---- , and $\cdots . .=7,16$, and $22 \mathrm{~h}$ ) and two light quantities ( $\square$ and $\Delta=5$ and $20 \mathrm{~mol}$ quanta $\left.\mathrm{m}^{-2}\right)$. assumed that one lesion could be approximated by one unit of colonized leaf area and have defined the basic reproductive number using the sporulation data by McGregor and Manners (7).

In another study Sache and de Vallavieille-Pope (11) found that single-spore inoculations led to medium density of infections due to the semisystemic nature of yellow rust; sporulation was estimated per lesion, even though the actual average area of one lesion is not provided. In addition to these differences between the two studies' method of inoculation, type of leaf area used as reference unit for the spore counts, and length of the sporulating period, the controlled environment conditions also differed. The day length was $2 \mathrm{~h}$ longer and the light quantity used was almost double that used by McGregor and Manners (7). However, even though the sporulation curves are not directly comparable, their shapes are very similar. It seems logical to assume that if the sporulation data we used referred to an average sporulating lesion, the difference in the final results would have been quantitative and not qualitative.

The method presented here for calculating the exponential growth rate, $r$, from the Euler equation as a function of weather variables and its sensitivity analysis, can be used for other types of sporulation curve. Segarra et al. (14) showed that the Vanderplank model is a special case of the Kermack and McKendrick model. In the case of the Vanderplank model the Euler equation (4) becomes

$$
1=R_{0} \frac{1}{i}\left[e^{-r(p+i)}-e^{-r p}\right]
$$

where $r$ is the exponential growth rate, $p$ is the latent period, and $i$ is the infectious period. All derivatives in equation 4 can easily be calculated for the Vanderplank model.

Our method can also be used to study the effects of the application of fungicides on the rate of epidemic progress. Different timings of applications and different types of fungicides can easily be investigated for their effect on epidemic progress. A protective fungicide would affect infections and hence, the basic reproductive number. A curative fungicide would delay sporulation, and hence, would affect the latent period and the mean of the sporulation curve. Different timings could further affect the results depending on weather conditions. Our results for yellow rust suggest that a fungicide targeting the basic reproductive number would be the best choice, especially at the beginning of the epidemic season (March/April) when temperatures are still quite low.

In this paper we have presented a method of sensitivity analysis for the exponential rate of epidemic growth. This method is easy to apply, can easily accommodate new information, and can provide insight into the relative importance of climatic variables and life cycle components for the epidemic growth rate.

\section{APPENDIX 1}

Derivation of the Euler equation. The basic reproductive number, $R_{0}$, is a key variable used in both plant and animal disease epidemiology and is defined as the total number of daughter infections (lesions) produced by a single mother infection (lesion) throughout the latter's infectious period at very low disease density (10). Assume that the rate of increase of the density of lesions is given by

$$
\frac{d Y(t)}{d t}=b(t)
$$

where $Y(t)$ is the number of pathogen lesions at time $t$ and $b(t)$ is the rate of appearance of new lesions per time unit $t$.

The age of infection of a lesion is the number of days elapsed since the lesion was caused (i.e., since the infection took place). The number of spores produced per unit time by a lesion of age of infection, $\tau$, is denoted by $I(\tau)$. Integration over the age of 
infection gives the total number of spores, $\Omega$, produced by one lesion during its entire infectious period (during the latent period $I(\tau)=0)$. The ratios of $I(\tau)$ and $\Omega$ give the probability distribution function, $i(\tau)$, which is called the sporulation curve. So

$$
i(\tau)=\frac{I(\tau)}{\int_{0}^{\infty} I(\tau) d \tau} \text { or } I(\tau)=\Omega i(\tau)
$$

In the application presented here, the gamma probability distribution function was the best choice for the sporulation data of McGregor and Manners (7). The fitted curves represent $I(\mathrm{t})$ (Fig. 1). The mean, $\mu$, and standard deviation, $\sigma$, of the gamma distribution are functions of the distribution parameters $n$ and $\lambda$ with $\mu=n / \lambda$ and $\sigma^{2}=n / \lambda^{2}$. Using nonlinear least square regression we obtained the fitted curves by comparing optimization results for $\lambda$ with $n$ set to different values. We got the best overall fit to the data by setting $n=3$ ( $R^{2}$ ranged from 79 to $93.4 \%$ ).

The rate of appearance of new lesions per time unit, $b(t)$, is assumed to be linearly dependent on the density of spores, $\Lambda(t)$, in the crop and the density of the healthy (susceptible) sites at time $t, H(t)$. So

$$
b(t)=\theta \Lambda(t) H(t)
$$

where $\theta$ is the probability that a spore lands on a healthy plant and causes an infection. The density of spores, $\Lambda(t)$, is the sum of all spores produced by the lesions in the crop. At time $(t-\tau), b(t-\tau)$ lesions were caused, each of which will produce $\Omega i(\tau)$ spores. To account for all ages of lesions present, integrate over all ages of infection to yield

$$
\Lambda(t)=\int_{0}^{\infty} b(t-\tau) \Omega i(\tau) d \tau
$$

Therefore in the initial stages of an epidemic, we have

$$
\frac{d Y(t)}{d t}=\Omega \theta H(t) \int_{0}^{\infty} b(t-\tau) i(\tau) d \tau
$$

At low pathogen density we assume that disease starts with as little as a single sporulating lesion producing $\Omega$ spores during its infectious period, the initial density of healthy sites is high, $H(t) \approx H_{0}$, and $R_{0}=\Omega \theta H_{0}$. At low pathogen density disease grows exponentially and if the initial disease level is $\mathrm{Q}$, then $Y(t)=\mathrm{Q} e^{\mathrm{rt}}$. Substituting the latter equations in A1 and A5 gives

$$
\begin{aligned}
& r Q e^{r t}=R_{0} \int_{0}^{\infty} r Q e^{r(t-\tau)} i(\tau) d \tau \Rightarrow \\
& 1=R_{0} \int_{0}^{\infty} e^{-\tau \tau} i(\tau) d \tau=1
\end{aligned}
$$

This is the Euler equation, which can be solved numerically to estimate $r$ if $i(\tau)$ and $R_{0}$ are known.

It is now easy to derive equation 2 in the main text from equation A6 using the fact that $i(\tau)$ is represented by a gamma probability density function with parameters $n$ and $\lambda$. So

$$
\begin{aligned}
& \int_{0}^{\infty} e^{-\tau \tau} i(\tau) d \tau=\int_{0}^{\infty} \frac{e^{-\tau \tau} \lambda^{n}(\tau-p)^{(n-1)} e^{\lambda(\tau-p)}}{\Gamma(n)} d \tau= \\
& \frac{\lambda^{n}}{\Gamma(n)} \int_{0}^{\infty} e^{-\tau \tau}(\tau-p)^{(n-1)} e^{\lambda(\tau-p)} d \tau= \\
& \frac{\lambda^{n}}{\Gamma(n) e^{r p}} \int_{0}^{\infty} e^{-\tau \tau} e^{r p}(\tau-p)^{(n-1)} e^{\lambda(\tau-p)} d \tau= \\
& \frac{\lambda^{n}}{\Gamma(n) e^{r p}(\lambda+r)^{n}} \int_{0}^{\infty} e^{-(\lambda+r)(\tau-p)}[(\lambda+r)(\tau-p)]^{(n-1)}(\lambda+r) d \tau= \\
& \frac{\lambda^{n}}{\Gamma(n) e^{r p}(\lambda+r)^{n}} \int_{0}^{\infty} e^{-(\lambda+r)(\tau-p)}[(\lambda+r)(\tau-p)]^{(n-1)} d[(\lambda+r)(\tau-p)]= \\
& \frac{\lambda^{n}}{\Gamma(n) e^{r p}(\lambda+r)^{n}} \Gamma(n)=\left(\frac{\lambda}{\lambda+r}\right)^{n} e^{-r p}
\end{aligned}
$$

$$
\mu=\frac{n}{\lambda} \Rightarrow n=\mu \lambda
$$

$$
\begin{gathered}
\sigma^{2}=\frac{n}{\lambda^{2}} \Rightarrow\left(\text { from A8) } \sigma^{2}=\frac{\mu \lambda}{\lambda^{2}}=\frac{\mu}{\lambda} \Rightarrow \lambda=\frac{\mu}{\sigma^{2}}\right. \\
\text { and } n=\left(\frac{\mu}{\sigma}\right)^{2}
\end{gathered}
$$

So equation A7 becomes

$$
\int_{0}^{\infty} e^{-\tau \tau} i(\tau) d \tau=\left(\frac{\frac{\mu}{\sigma^{2}}}{\frac{\mu}{\sigma^{2}}+r}\right)^{n} e^{-r p}
$$
After dividing equation A10 with $\frac{\mu}{\sigma^{2}}$ we get equation 2 in the
main text.

\section{APPENDIX 2}

Derivation of equation 4 in the main text. Equation 3 cannot be solved for $r$ explicitly. We do, however, want to calculate the sensitivity of $r$ to $x, \partial r / \partial x$. Equation 3 can be written as

$$
G\left[r(x), R_{0}(x), p(x), \mu(x), \sigma(x)\right]=1
$$

Taking the derivative of $G$ with respect to $x$ gives equation A12

$$
\left(\frac{\partial G}{\partial r} \frac{\partial r}{\partial x}\right)+\left(\frac{\partial G}{\partial R_{0}} \frac{\partial R_{0}}{\partial x}\right)+\left(\frac{\partial G}{\partial p} \frac{\partial p}{\partial x}\right)+\left(\frac{\partial G}{\partial \mu} \frac{\partial \mu}{\partial x}\right)+\left(\frac{\partial G}{\partial \sigma} \frac{\partial \sigma}{\partial x}\right)=0
$$

Solving equation A12 for $\partial r / \partial x$ gives

$$
\begin{aligned}
& \frac{\partial r}{\partial x}= \\
& -\left(\frac{\partial G}{\partial r}\right)^{-1}\left[\left(\frac{\partial G}{\partial R_{0}} \frac{\partial R_{0}}{\partial x}\right)+\left(\frac{\partial G}{\partial p} \frac{\partial p}{\partial x}\right)+\left(\frac{\partial G}{\partial \mu} \frac{\partial \mu}{\partial x}\right)+\left(\frac{\partial G}{\partial \sigma} \frac{\partial \sigma}{\partial x}\right)\right]= \\
& -\left[\left(\frac{\partial G}{\partial r}\right)^{-1} \frac{\partial G}{\partial R_{0}}\right] \frac{\partial R_{0}}{\partial x}-\left[\left(\frac{\partial G}{\partial r}\right)^{-1} \frac{\partial G}{\partial p}\right] \frac{\partial p}{\partial x} \\
& -\left[\left(\frac{\partial G}{\partial r}\right)^{-1} \frac{\partial G}{\partial \mu}\right] \frac{\partial \mu}{\partial x}-\left[\left(\frac{\partial G}{\partial r}\right)^{-1} \frac{\partial G}{\partial \sigma}\right] \frac{\partial \sigma}{\partial x}
\end{aligned}
$$

All derivatives in this expression can be calculated from equation 3 in the main text and from the equations in Table 1. For ease of interpretation, the expressions in square brackets are rewritten to give equation 4 in the main text, i.e.,

$$
\frac{\partial r}{\partial x}=-\left[\left(\frac{\partial r}{\partial R_{0}} \frac{\partial R_{0}}{\partial x}\right)+\left(\frac{\partial r}{\partial p} \frac{\partial p}{\partial x}\right)+\left(\frac{\partial r}{\partial \mu} \frac{\partial \mu}{\partial x}\right)+\left(\frac{\partial r}{\partial \sigma} \frac{\partial \sigma}{\partial x}\right)\right]
$$

The epidemiological variables $R_{0}, r, p, \sigma$, or $\mu$ can be functions of more than one weather variable and they might also not all depend on the same set of weather variables. The case showed above is for the simple case of dependence of all of these epidemiological variables on a common weather variable, $x$, which is what applies when the sensitivity of the exponential growth rate to temperature is investigated in the main text. In the case of dependence of some of the epidemiological variables, apart from $r$, on a weather variable, $x$, the terms in the sum above relating to the epidemiological variables not dependent on $x$ will 
be replaced by zero. So, if only $R_{0}$, apart from $r$, are functions of $x$, the sensitivity of $r$ to $x$ is given by

$$
\frac{\partial r}{\partial x}=-\left[\left(\frac{\partial r}{\partial R_{0}} \frac{\partial R_{0}}{\partial x}\right)+0+0+0\right]
$$

This applies when the sensitivity of the exponential growth rate to dew period or light quantity is investigated in the main text.

\section{ACKNOWLEDGMENTS}

This project has been funded by The Department for Environment, Food and Rural Affairs (DEFRA). Rothamsted Research receives support from The Biotechnology and Biological Sciences Research Council (BBSRC), Swindon, United Kingdom.

\section{LITERATURE CITED}

1. Caswell, H. 2001. Matrix Population Models: Construction, Analysis and Interpretation. Sinauer Associates Inc., Sunderland, MA.

2. Chen, X. M. 2005. Epidemiology and control of stripe rust [Puccinia striiformis f. sp. tritici] on wheat. Can. J. Plant Pathol. 27:314-337.

3. de Vallavieille-Pope, C., Huber, L., Leconte, M., and Bethenod, O. 2002. Preinoculation effects of light quantity on infection efficiency of Puccinia striiformis and P. triticina on wheat seedlings. Phytopathology 92:1308-1314.

4. de Vallavieille-Pope, C., Huber, L., Leconte, M., and Goyeau, H. 1995. Comparative effects of temperature and interrupted wet periods on germination, penetration, and infection of Puccinia recondita f. sp. tritici and $P$. striiformis on wheat seedlings. Phytopathology 85:409-415.

5. Kolnaar, R. W., and van den Bosch, F. 2001. Effect of temperature on epidemiological parameters of Puccinia lagenophorae. Plant Pathol. 50:363370.

6. Madden, L. V., and Campbell, C. L. 1987. Potential effects of airpollutants on epidemics of plant diseases. Agric. Ecosyst. Environ. $18: 251-262$.

7. McGregor, A. J., and Manners, J. G. 1985. The effect of temperature and light intensity on growth and sporulation of Puccinia striiformis on wheat. Plant Pathol. 34:263-271.

8. Papastamati, K., van den Bosch, F., Welham, S. J., Fitt, B. D. L., Evans, N., and Steed, J. M. 2002. Modeling the daily progress of light leaf spot epidemics on winter oilseed rape (Brassica napus), in relation to Pyrenopeziza brassicae inoculum concentrations and weather factors. Ecol. Model. 148:169-189.

9. Paveley, N. D., Lockley, D., Vaughan, T. B., Thomas, J., and Schmidt, K. 2000. Predicting effective fungicide doses through observation of leaf emergence. Plant Pathol. 49:748-766.
10. Rapilly, F. 1979. Yellow rust epidemiology. Annu. Rev. Phytopathol. 17:59-73.

11. Sache, I., and de Vallavieille-Pope, C. 1993. Comparison of the wheat brown and yellow rusts for monocyclic sporulation and infections processes, and their polycyclic consequences. J. Phytopathol. 138:55-65.

12. Salas, S. L. 1995. Salas and Hille's Calculus: One and Several Variables. 1st ed. John Wiley \& Sons, NY.

13. Scott Mills, L., Doak, D. F., and Wisdom, M. J. 1999. Reliability of conservation actions based on elasticity analysis of matrix models. Conserv. Biol. 13:815-829.

14. Segarra, J., Jeger, M. J., and van den Bosch, F. 2001. Epidemic dynamics and patterns of plant diseases. Phytopathology 91:1001-1010.

15. Singh, R. P., Huerta-Espino, J., and Roelfs, A. P. 2002. The wheat rusts. Pages 227-249 in: Bread Wheat: Improvement and Production. B. C. Curtis, S. Rajaram, and H. Gómez-Macpherson, eds. Food and Agriculture Organization (FAO) Plant Production and Protection Series No. 30, FAO of the United Nations.

16. van den Bosch, F., Frinking, H. D., Metz, J. A. J., and Zadoks, J. C. 1988. Focus expansion in plant disease: III. Two experimental examples. Phytopathology 78:919-925.

17. van den Bosch, F., Verhaar, M. A., Buiel, A. A. M., Hoogkamer, W., and Zadoks, J. C. 1990. Focus expansion in plant disease: IV. Expansion rates in mixtures of resistant and susceptible hosts. Phytopathology 80:598-602.

18. van den Bosch, F., Zadoks, J. C., and Metz, J. A. J. 1988. Focus expansion in plant disease II: Realistic parameter-sparse models. Phytopathology 78:59-64.

19. Vanderplank, J. E. 1963. Plant Disease: Epidemics and Control. Academic Press, NY.

20. van Oijen, M. 1992. Selection and use of a mathematical model to evaluate components of resistance to Phytophthora infestans in potato. Neth. J. Plant Pathol. 98:192-202.

21. Viljanen-Rollinson, S. L. H., Marroni, M. V., Butler, R. C., Deng, Y., and Armour, T. 2005. Latent periods of Septoria tritici blotch on ten cultivars of wheat. N.Z. Plant Prot. 58:256-260.

22. West, J. S., Kharbanda, P. D., Barbetti, M. J., and Fitt, B. D. L. 2001. Epidemiology and management of Leptosphaeria maculans (phoma stem canker) on oilseed rape in Australia, Canada, and Europe. Plant Pathol. 50:10-27.

23. Xu, X.-M., Butt, D. J., and van Santen, G. 1995. A dynamic model simulating infection of apple leaves by Venturia inaequalis. Plant Pathol. 44:865-876.

24. Young, C. S., Paveley, N. D., Vaughan, T. B., Thomas, J. M., and Lockley, K. D. 2003. Predicting epidemics of yellow rust (Puccinia striiformis) on the upper canopy of wheat from disease observations on lower leaves. Plant Pathol. 52:338-349.

25. Zadoks, J. C. 1961. Yellow rust on wheat: Studies in epidemiology and physiologic specialization. Tijdschr. over Plantenziekten 67:69-256.

26. Zadoks, J. C. 1971. Systems analysis and dynamics of epidemics. Phytopathology 61:600-610. 